Relations industrielles

Industrial Relations

\title{
Démocratie ouvrière en 1952
}

\section{Emile Gosselin}

Volume 8, numéro 1, décembre 1952

URI : https://id.erudit.org/iderudit/1022974ar

DOI : https://doi.org/10.7202/1022974ar

Aller au sommaire du numéro

Éditeur(s)

Département des relations industrielles de l’Université Laval

ISSN

0034-379X (imprimé)

1703-8138 (numérique)

Découvrir la revue

Citer cet article

Gosselin, E. (1952). Démocratie ouvrière en 1952. Relations industrielles / Industrial Relations, 8(1), 45-67. https://doi.org/10.7202/1022974ar
Résumé de l'article

Dans une première section, l'auteur fait un bref commentaire sur la nature et l'importance des congrès annuels des mouvements ouvriers canadiens et sur l'action politique de ces derniers. Le lecteur trouvera dans la deuxième partie du travail un compte-rendu des discours, rapports et résolutions présentés lors de ces réunions.
Tous droits réservés (C) Département des relations industrielles de l’Université Laval, 1952
Ce document est protégé par la loi sur le droit d'auteur. L’utilisation des services d'Érudit (y compris la reproduction) est assujettie à sa politique d'utilisation que vous pouvez consulter en ligne.

https://apropos.erudit.org/fr/usagers/politique-dutilisation/ 


\section{Démocratie ouvrière en 1952}

\section{Emile Gosselin}

Dans une première section, lauteur fait un bef commentaire sur la nature et l'importance des congrès annuels des mouvements ouvriers canadiens et sur l'action politique de ces derniers. Le lecteur trouvera dans la deuxième partie du travail un compte-rendu des discours, rapports et résolutions présentés lors de ces réunions.

\section{I-LES CONGRES OUVRIERS}

Les trois grandes centrales ouvrières du Canada viennent de tenir leur congrès annuel au cours des mois d'aoùt et de septembre. Du 18 au 23 août, 537 délégués du Congrès des Métiers et du Travail, représentant plus d'un demi-million de syndiqués (523,000 environ) se réunissaient à Winnipeg sous la présidence de $\mathrm{M}$. Percy R. Bengough. C'était la cinquième fois que cette prodigieuse cité de l'ouest canadien recevait les délégués de notre plus grande centrale ouvrière. Le deuxième congrès, tenu du 14 au 18 septembre, sous la présidence de M. Gérard Picard, réunissait à Shawinigan Falls les 400 délégués de la Confédération des travailleurs catholiques du Canada, groupant 95,000 membres, soit le mouvement ouvrier le plus important de notre Province. Enfin la Ville Reine accueillait les 934 délégués du Congrès Canadien du Travail, la délégation la plus importante de tous les congrès tenus cette année. Il est à noter que c'est à Toronto même que, en septembre 1940, le Congrès Canadien du Travail prit naissance. C'était donc avec une vive satisfaction que le président, Monsieur A. $\mathrm{R}$. Mosher, pouvait regarder le chemin parcouru par la centrale ouvrière qui se place maintenant au deuxième rang parmi nos mouvements, avec au-dela de 330,000 membres.

GOSSELIN, EmILe, licencié en droit (Montréal), M.A. en économique (Toronto), secrétaire du Département de relations industrielles de Laval. chargé du cours d'économique du travail et directeur des recherches en relations industrielles. 


\section{Importance des Congrès}

Les congrès de nos centrales ouvrières ont pour buts généraux d'éclairer et d'orienter les délégués en plus de leur permettre de participer de façon démocratique à la vie du mouvement syndical. Un congrès permet aux membres de renforcer le lien de solidarité qui les unit dans la poursuite des objectifs communs. Au cours de ces rencontres, on discute des problèmes d'organisation surgissant dans différentes régions du pays et on donne au mouvement son orientation véritable. Les principes sont remis en lumière de même que les techniques d'action sont soumises à de profonds examens. C'est également au cours de ces réunions annuelles que l'on peut faire la mise au point sur les positions du travail organisé à l'endroit des problèmes économiques, politiques et sociaux qui affectent le monde ouvrier et qui ne sont au fond que des aspects très importants de la vie nationale elle-même.

C'est avec raison que la presse, la radio et les agences de nouvelles décrivent, commentent ou font la critique des diverses phases de ces réunions, car le mouvement syndical se rattache très intimement au mouvement de progression du pays lui-même. On ne peut traiter du problème syndical en vase clos. Et si l'on fait l'analyse des discours et des débats qui ont lieu à ces occasions, on remarque le souci d'attirer le public tout entier, citoyens comme gouvernants, au coeur même des problèmes à l'étude. En résumé les Congrès s'adressent à la fois aux membres des centrales ouvrières de même quaux citoyens du pays ou de la province. C'est donc parmi un auditoire beaucoup plus vaste que celui composé par les seuls délégués que les débats sur les problèmes du travail s'engagent véritablement.

\section{Action politique}

Chaque année les centrales ouvrières du pays font connaître aux différents gouvernements leurs points de vue sur certains aspects de la vie politique provinciale ou nationale et elles exercent des pressions pour que ces gouvernements adoptent des programmes conformes aux meilleurs intérêts de la classe ouvrière. Cependant beaucoup de travailleurs, en particulier les membres des organisations ouvrières, en sont venus à la conclusion que l'orientation politique restreinte à l'éducation civique seule ou aux simples présentations par les syndicats de leurs griefs ou de leurs revendications ne donnait pas les résultats espérés, parce que ceux-ci sont trop lents à venir, alors que les problèmes du travail sont urgents. L'attitude présente du monde syndical est d'exercer à l'avenir, par des méthodes appropriées et conformes à un véri- 
table syndicalisme agissant dans l'Etat moderne, une influence beaucoup plus directe sur le public et les gouvernants au moyen de l'Action politique directe non-partisane. Le fait n'est pas nouveau dans nos annales syndicales, mais deux congrès, celui du C.C.T. et de la C.T.C.C. l'ont affirmé cette année avec une telle force et une telle conviction, que l'on peut entrevoir de profonds changements dans l'orientation de tous nos mouvements ouvriers.

\section{L'opinion des Congrès sur l'action politique}

Monsieur Mosher, du C.C.T., dans son discours présidentiel à Toronto disait: «Je voudrais réaffirmer ma conviction que le prochain grand pas du mouvement syndical au Canada doit se faire dans le domaine de la politique. Nous n'avons pas commencé à réaliser toutes les possibilités de notre force en politique, et, par conséquent, les intérêts des travailleurs, pour autant qu'il s'agisse de la législation ouvrière, n'ont pas reçu cette attention à laquelle ils ont droit».

Plus loin dans son discours le président indique quelques raisons qui selon lui justifie l'action politique syndicale: «Les vieux partis politiques affirment qu'ils représentent le peuple, mais en réalité ils sont responsables vis-à-vis les intérêts privés qui leur fournissent des fonds électoraux et contrôlent les décisions politiques. La seule façon pour les travailleurs de remédier à cet état de choses est de s'organiser dans le domaine politique, et d'élire leurs propres représentants tant au Parlement qu' aux législatures. »

Reprenant ce problème dans son rapport moral, monsieur Gérard Picard, président de la Confédération des Travailleurs Catholiques, déclarait devant les 400 délégués réunis à Shawinigan: "Dans la poursuite de son but qui est, en termes généraux, l'étude, la défense et la promotion des intérêts professionnels, économiques et sociaux de ses membres, une organisation syndicale de travailleurs a le droit de recourir à tous les moyens honnêtes qui lui permettront de l'atteindre. Il s'agit d'éviter, c'est élémentaire, que le recours à un moyen, même honnête, ne la détourne de sa fin. »

«Il n’y a pas de nécessité absolue, pour une organisation syndicale de travailleurs, de faire de l'action politique. Mais lorsque la législation du travail et son application deviennent une source d'injustices, lorsque des gouvernements se montrent hostiles aux syndicats de travailleurs ou supportent si mal la contradiction qu'ils cherchent constamment une occasion d'exercer des représailles contre des dirigeants syndicaux, l'action politique peut devenir nécessaire, de nécessité de moyen, pour aler- 
ter l'opinion publique. Qu'y a-t-il d'antidémocratique en cela ? Et même si, au cours d'une campagne électorale, l'action politique des syndicats conduit à des déclarations jugées inopportunes, voire un peu osées, ce pourrait être une raison suffisante pour des rappels à l'ordre, mais non une raison suffisante pour écarter l'action politique comme recours des syndicats lorsque les autres moyens n'ont pas donné de résultats satisfaisants. »

Cette déclaration s'éclaire si on la rapproche d'une réflexion que fait M. Picard à un autre moment de son discours présidentiel: «l'action politique des syndicats de travailleurs diminue d'intensité lorsqu'il existe un climat de confiance et que la législation du travail est préparée et appliquée suivant la formule tripartite qui prévoit la collaboration de l'Etat, des associations patronales et des syndicats de travailleurs. Comme l'ont fait observer, dès 1919 , les fondateurs de l'Organisation Internationale du Travail:

«La vie sociale ne peut pas et ne doit pas être régie par les gouvernements dans la solitude orgueilleuse, mais stérile, de leur souveraineté. »

«Chez nous, le climat de confiance n'existe pas et la situation est tendue depuis plusieurs années. De plus la formule tripartite n'est pas acceptée, dans le concret, pour assurer la préparation et l'application de la législation du travail. Peut-on remédier à cet état de choses? Ce devrait être encore plus possible dans une province catholique quailleurs. Pourquoi n'y arrive-ton pas? Prenons deux ou trois cas entre cent, et nous devrions finir par nous comprendre. »

La nouvelle orientation politique syndicale n'est pas qu'un simple geste de défense contre des actes contraires au syndicalisme lui-même. Cette action politique, selon MM. Mosher et Picard, semble conforme aux meilleurs intérêts du syndicalisme et des travailleurs et repose sur les exigences mêmes du mouvement et de ses aspirations. Citons de plus ici M. le Chanoine Henri Pichette, aumônier général de la C.T.C.C. Après avoir demandé à l'assemblée si elle doit se désintéresser de la politique «au point de nous limiter à des biens particuliers sans voir le bien de toute la société? 》, l'aumônier général poursuit: "Ce serait faux et nous aurions tort, sous prétexte que c'est très délicat de l'ignorer. Le problème social en est un de relation entre les individus et toutes les classes de la société et il nous appartient d'apporter notre contribution. Notre syndicalisme a un objet qui est d'ordre professionnel, économique. 
C'est son objet propre mais il n'est pas limité, isolé du reste, et on ne peut le détacher de tous les autres problèmes que présente la société sans risquer de le fausser. Si nous sommes une partie d'un ensemble et que nous devons former avec les autres cet ensemble, nous ne pouvons ignorer le dernier et nous en séparer sous prétexte que ce n'est pas notre domaine. Il vous appartient donc de faire de l'action politique car autrement vous ne seriez pas les premiers artisans de votre propre restauration et de votre intégration ou vous vous exposeriez à être ignorés des autres. Il vous appartient donc en même temps de considérer toutes les circonstances dans lesquelles votre action politique doit s'effectuer. Vous ne pouvez ignorer le passé. Vous ne pouvez méconnaître l'état des esprits, mais vous ne pouvez en même temps renoncer aux responsabilités qui vous incombent, de collaborer avec les autres sur le plan politique à bien situer et à intégrer la classe des travailleurs. Dans cette tâche, vous contribuerez à redonner à la politique toute la noblesse qu'elle doit avoir. $\triangleright$

\section{Action politique énoncée aux congrès}

C.C.T.:

Le C.C.T. prépare un programme politique et le soumet à tous les partis politiques. Il ne s'affilie à aucun parti, mais il «endosse» momentanément ce parti et les candidats qui acceptent le programme politique du mouvement. Il entend rester indépendant de tout parti, même si à un moment donné il accorde en tant que mouvement un support formel à un parti ou à des candidats. Il cherche à stimuler les candidatures politiques parmi les ouvriers et il accepte que des membres assument de leur propre gré des postes de commande dans un parti. Présentement le C.C.T. endosse la politique du parti C.C.F. et recommande aux membres de le supporter.

\section{C.M.T.C.:}

Rien n'indique dans les rapports parvenus jusqu'ici que le sujet ait été abordé cette année de la façon que l'on fait le C.C.T. et la C.T.C.C. Rappelons cependant la politique traditionnelle de ce mouvement de laisser pleine liberté aux syndicats eux-mêmes et de favoriser une politique non-partisane décentralisée à l'échelon local ou provincial. La centrale favorise le développement de comités politiques locaux ou provinciaux. A l'occasion les syndicats favoriseront des mesures ou des candidats favorables au mouvement. 
C.T.C.C.:

\section{a) La déclaration de M. Picard au Congrès}

«Au cours des derniers mois, l'on a semé tellement de confusion sur l'attitude de la C.T.C.C. qu il importe de clarifier une fois pour toute la situation. La C.T.C.C. n'a pas fondé et ne songe nullement à fonder un parti politique. Il n'y a aucun lien organique entre aucun parti politique et la C.T.C.C. Par voie de conséquence, cependant, l'action politique, au sein de la C.T.C.C., a pu favoriser et a, de fait, favorisé certains candidats d'un parti politique, comme elle a causé la défaite de certains candidats d'un autre parti politique.» Plus loin il ajoute: «Il ne faudrait pas conclure de ce qui précède ${ }^{1}$ que la C.T.C.C. tient à s'engager à fond dans l'action politique directe et non-partisane. Mais la C.T.C.C., sans aller aussi loin que la plupart des centrales syndicales de travailleurs des autres pays démocratiques, a considéré qu'elle devait s'engager sur la voie de l'action politique, sans toutefois lier son sort à celui d'aucun parti politique. »

Après avoir fait de nombreuses suggestions concernant un comité de relations industrielles à l'Assemblée législative et une liste permanonte de tiers-arbitre, M. Picard poursuivait: «il semble que la C.T.C.C. devrait maintenir son Comité d'orientation politique et favoriser la formation de comités régionaux et locaux agissant de concert avec le comité provincial ». Un sujet comme la revision de la carte électorale de la Province de Québec pourrait être abordée par tous les comités en même temps.

\section{b) Résolutions}

Les membres délégués ont adopté des résolutions dont voici quelques points importants:

1) Le Comité d'Orientation Politique fera connaître «dans le journal Le Travail les attitudes favorables ou non des hommes politiques à l'égard des travailleurs ».

2) A l'avenir le journal Le Travail refusera de publier toute annonce des partis politiques.

3) Adoption de la résolution du Bureau Confédéral ${ }^{2}$ sur l'action politique, en date du 27 mars 1952, où l'on déclare entre autre:

(1) De l'Action politique antérieure au sein de la C.T.C.C.

(2) Voir cette résolution dans la section «informations». 
«A) La C.T.C.C., comme telle, n'appuiera aucun candidat, ni aucun parti politique;

«B) Les officiers et membres du Bureau confédéral, à J'exception du président général et du secrétaire général qui, à cause de leurs fonctions, engagent plus directement la C.T.C.C., seront libres d'exercer tous les droits que leur confère leur titre de citoyen; 》

Au cours du Congrès, M. Gérard Picard précisa par ailleurs l'intention de cette résolution en deux points: 1 ) «que le président général et le secrétaire général ne posent pas leur candidature sans démissionner de leur poste; 2 ) qu ils ne participent à aucune assemblée politique convoquée par les partis politiques. »

4) Approbation du rapport du président général et du Comité d'orientation politique, sur les questions touchant l'Action politique.

\section{Conclusions}

Sans juger ici des méthodes politiques utilisées dans le monde syndical, on peut dire au moins que l'orientation générale des syndicats dans la sphère politique répond non seulement à la réalité des faits mais elle confirme la nouvelle conception qui anime le mouvement syndical d'aujourd'hui. Le syndicalisme est né à un moment où la théorie politique proclamait que le mouvement n'était acceptable que s'il se limitait à améliorer le sort du travailleur par la négociation collective, avec usage, à l'occasion du droit de grève. Mais cette théorie semble aujourd'hui très incomplète, même si elle souligne deux des conditions essentielles à la survie et au progrès d'un syndicalisme libre et agissant.

Laction gouvernementale affecte constamment le travailleur et son syndicat dans des matières qui dépassent les cadres de la convention collective et qui ne peuvent être étudiées à leur juste mérite que sur le plan politique. En réalité, la vie économique et sociale dans laquelle évolue le travailleur moderne est si complexe et interdépendante, que tout ce qui est politique l'affecte. ${ }^{3}$

Pour autant il est nécessaire que les mouvements ouvriers revendiquent les droits des travailleurs et les défendent dans l'arène politique elle-même, en employant ces techniques les mieux appropriées à la

(3) Nous discuterons plus à fond de l'brientation du syndicalisme dans le domaine politique au cours d'un prochain numéro de la revue. 
poursuite du bien général et à la protection des intérêts collectifs et individuels des travailleurs.

\section{II-SYNOPSIS DES CONGRES OUVRIERS}

\section{Communisme}

Les trois congrès ont réitéré leur opposition indomptable au communisme. Monsieur Mosher, président du C.C.T. s'exprinait en ces termes: «Le Congrès Canadien du Travail a non seulement coopéré dans la lutte contre le communisme dans le domaine international, mais il a également été très agissant dans la lutte contre le communisme au Canada même. Comme vous le savez tous, le Congrès a expulsé les syndicats affiliés à trois unions internationales dominées par des communistes bien connus; de plus, il a accordé son appui constant à des syndicats reconnus dans les industries où les syndicats dominés par les communistes avaient été organisés. - Dans ces circonstances, il n'y a aucune raison pour qu'un travailleur canadien, quelqu'il soit, appartienne à un syndicat communiste. »

La question du communisme souleva un débat très animé lors de la réunion du C.M.T.C. Le président, M. Percy Bungough fut très véhément: «Nous avons nos réactionnaires qui travaillent pour un système de gouvernement qui base son économie sur le travail d'esclavage. Il y a trop longtemps que ces communistes et leurs adeptes qui essayent de nous réduire à l'esclavage posent en libérateurs. Le temps est venu où ce Congrès comme partie du mouvement Trade-Unioniste libre doit exposer leur trahison et leur duplicité. Ce Congrès en tant que partie importante de la Confédération internationale des unions ouvrières libres... ne peut pas en toute sincérité faire autre chose que de les éliminer de ses propres organisations au pays.» Et plus loin il ajoute. «Il ne serait ni consistent, ni sensible de dépenser tant d'énergie et de ressources financières pour combattre l'expansion du communisme à l'extérieur et, en même temps, dorloter et soigner les adeptes de cette même philosophie dans nos organisations et notre pays.» Le Congrès ne fut cependant pas unanime sur les méthodes à employer pour combattre ce danger. Les délégués rejetèrent une résolution ayant l'appui du bureau du C.M.T.C. et demandant l'interdiction du parti communiste, du parti ouvrier progressiste et de «toute autre organisation visant la destruction de notre genre de vie démocratique. » A l'encontre de cette résolution, certains orateurs déclarèrent que si un gouvernement s'engageait dans cette voie, personne ne saurait « où il s'arrêtera, ni qui sera le suivant. » Un autre déclara: «Vous ne pouvez légiférer 
sur les idées politiques... Rien ne prouve qu en mettant une chose hors la loi on y met fin. » Le Congrès vota cependant contre une résolution demandant d'abroger les articles de la constitution interdisant l'affiliation des syndicats dominés par des communistes. Si le C.M.T.C. hésitait à demander la mise hors la loi du parti communiste, il entendait bien procéder avec une extrème rigueur contre les partisans de cette doctrine au sein même de son organisation.

La C.T.C.C. est opposée par principe au communisme. Le président, M. Gérard Picard, non moins que M. Jean Marchand, le secrétaire de la Confédération et les délégués s'indignèrent contre certaines insinuations à l'égard de la position de leur mouvement vis-à-vis le communisme. "Lion tente de faire croire que la C.T.C. serait une alliée du communisme. Inutile de dire que cette campagne de dénigrement est aussi fausse que ridicule. Il est évident que cette campagne de dénigrement a pour but de jeter du doute dans les esprits et surtout d'atteindre la réputation des dirigeants actuels du mouvement. »

«Parce qu'il y a une église catholique à Moscou, va-t-on prétendre que le Vatican est l'alliée du Kremlin ?...» Avec une fine ironie, le président dispose de nombreuses objections et finalement il explique la position de son mouvement. «La C.T.C.C. est opposée au communisme par conviction, non par peur. Elle est opposée au communisme parce que le communisme est athé et qu'il n'a aucun respect pour la personne humaine et la famille. La C.T.C.C. s"inspire de la doctrine sociale de l'Eglise et place les valeurs spirituelles au premier rang dans la hiérarchie des valeurs. »

Dans le Rapport du Bureau Confédéral, M. Jean Marchand reprend le même thème et déclare que les syndicats catholiques «sont devenus les ennemis numéro 1 de tous les tenants, conscients et inconscients, du libéralisme économique.

«Car, ne l'oublions pas, pour ces gens, le bien suprême est l'argent et, tous ceux qui menacent leurs intérêts sont des êtres dangereux à être classés dans la même catégorie. Ils ne combattent pas le communisme parce que le système qu'il veut implanter avilit la personne humaine et nie l'existence de Dieu mais exclusivement parce qu'il constitue un danger pour leurs intérêts immédiats. Nous croyons que si nous pouvions leur démontrer que le communisme est une idée rentable, ils en favoriseraient l'expansion. » 
La C.T.C.C. réitère donc sa soumission à la doctrine sociale de l'Eglise et entend poursuivre sa tâche économique et sociale malgré que ce mouvement «né et se développant dans un milieu catholique, rencontre autant d'opposition, soit victime d'autant de vexations et ait autant de difficultés à s'intégrer dans la société. »

\section{Cartel inter-syndical}

La formation d'un cartel inter-syndical en vue de présenter un front uni au moment des revendications ouvrières les plus importantes avait soulevé beaucoup d'enthousiasme il y a plusieurs mois dans les milieux syndicaux. Mais aujourd'hui le cartel est disparu, non par suite d'une mésentente sur la nécessité du cartel lui-même, mais à cause des divergences entre les membres du cartel sur leurs méthodes respectives d'action.

Dans son Rapport, M. Jean Marchand, secrétaire général de la C.T.C.C. déplore que le cartel inter-syndical «n'ait pu résister à certaines dissensions. Il est certainement dans le plus grand intérêt des travailleurs que les centrales syndicales présentent un front uni dans certaines circonstances particulières ». Soulignant la participation et l'aide reçue de la Fédération Provinciale et du Congrès Canadien lors d'une grève qui fit beaucoup de bruit dans nos milieux catholiques, M. Marchand indiquait avec à-propos la raison d'être de cette collaboration intersyndicale, « où il n'y aura plus moyen de diviser le travail organisé en se servant alternativement d'une centrale pour affaiblir l'autre au bénéfice d’intérêts étrangers. »

Monsieur Bengough du C.M.T.C. regrettait-il vraiment l'anéantissement du cartel par suite du refus de son mouvement d'en prolonger l'existence? Il affirme ouvertement du moins que le C.M.T.C. s'en retira à cause des actes du C.C.T. "Pendant un certain temps, j'ai cru que nous parvenions à quelque chose; mais les résultats ont été nuls. Bien que le comité semblât être une bonne affaire, je me suis opposé fortement au C.C.T., qui tentait de profiter de ce comité pour ruiner notre organisation. Il devint impossible de maintenir ce comité. » Un syndicat présenta une résolution demandant l'unité ouvrière, mais elle fut repoussée par les délégués à la suite de la sortie de $\mathrm{M}$. Bengough contre le C.C.T. A l'appui de la résolution visant à l'unité ouvrière, on allégua avec beaucoup de sens et de conviction que les organisations ouvrières n'avaient pas tout le succès désiré dans leurs revendications législatives, parce que chacune d'entre elles se présentait seule devant 
le gouvernement, alors les revendications des plus grandes organisations ouvrières étaient presquiidentiques. Il en résulte une grande confusion chez les travailleurs, parce que selon un orateur: «La masse des travailleurs ne peut pas comprendre pourquoi les groupes ouvriers ne travaillent pas conjointement. » Il semble bien, que le C.M.T.C. n'envisage pas pour l'instant de prendre l'initiative de réformer le cartel inter-syndical.

Il fut fortement, sinon violemment question, lors de la réunion du C.C.T., des attaques du C.M.T.C. à son égard. M. Donald MacDonald surtout se chargea de définir la position de la centrale dont il est secrétaire-trésorier, au sujet de l'Unité ouvrière. Il parla de rebuffades et d'insultes de la part du C.M.T.C., mais dit-il: « Nous sommes prêts et nous tenons à offrir notre amitié, mais nous refusons de trahir les intérêts de nos membres. Nous voulons être associés égaux.» Plus loin il poursuivait: «Nous avons fait notre possible pour recréer au Canada un seul organisme ouvrier, un congrès parlant d'une seule voix. » Malgré toutes les plaintes formulées par les délégués du C.C.T. à l'égard d'autres centrales ouvrières, en particulier du C.M.T.C., l'assemblée vota par une majorité écrasante une résolution recommandant au bureau de la centrale d'entrer en pourparlers avec le C.M.T.C. et la C.T.C.C. pour créer une unité d'action lorsqu'il s'agit de «toutes les questions intéressant les travailleurs du Canada ».

\section{Revendications syndicales}

Si le mouvement ouvrier du pays rencontre de réelles difficultés à former un cartel pour ses revendications législatives et autres, il faut y voir surtout un état temporaire de déséquilibre entre les centrales, situation causée par les circonstances particulières à des mouvements connaissant une expansion formidable. Les divergences semblent porter surtout sur les méthodes d'action et sur les champs respectifs d'organisation. Sur le fond même des problèmes auxquels ils font face, de même que sur les programmes de réforme, l'unanimité est presque complète dans tous les secteurs ouvriers.

\section{a) Assurance-santé}

Une résolution présentée aux délégués du C.M.T.C. charge l'Exécutif de cette centrale de continuer à faire pression pour obtenir un plan national d'assurance-santé lequel serait subsidié et contributoire. Il s'agirait d'un système «accordant soins médicaux, chirurgicaux, optiques, psychopatiques et dentaires, et les services appropriés d'hospitalisation et d'infirmières, à tous ceux qui en ont besoin », et dont les travailleurs accepteraient une juste proportion des frais. 
Les délégués au C.C.T. eurent à choisir entre différentes résolutions dans le sens de celle déjà adoptée par le C.M.T.C. On adopta, à l'unanimité, et sans discussion un voeu d'expression assez générale recommandant «un régime approprié de santé en conformité des demandes du travail organisé, de la Légion canadienne et d'autres groupes représentatifs de citoyens. »

\section{b) Pension de vieillesse}

Les délégués du C.M.T.C. demandent à l'Exécutif de continuer ses efforts dans le but d'obtenir des plans améliorés de vieil âge et de pension de retraite «en sorte de prévoir le versement d'une pension à l'âge de 65 ans pour les hommes et de 60 ans pour les femmes.»

\section{c) Impôts}

Les délégués aux trois congrès eurent à se prononcer sur des résolutions dont la teneur générale était à peu près la même.

C.M.T.C.:

Une résolution fusionnant tous les voeux exprimés demande au gouvernement, entre autres choses, que l'on puisse déduire jusqu'à concurrence de $10 \%$ du revenu, tous les frais médicaux de même que les traitements prescrits, y compris les soins de dentiste et d'opticien. Il en serait de même du coût, du prix d'achat et des frais d'entretien des outils nécessaires à l'exercice du métier. Egalement que le taux d'impôt soit gradué selon le revenu afin que le taux soit diminué pour les groupes recevant des revenus moins élevés. Que les exemptions d'impôt soient les suivantes: personnes mariées $\$ 3,000.00$; célibataires, $\$ 1,500.00$; Que l'exemption soit portée dę $\$ 250.00$ à $\$ 300.00$ dans le cas des femmes mariées dont le mari travaille; que les allocations familiales soient exemptées de l’impôt. Enfin, que le salaire de surtemps soit exempt d'impôt sur le revenu.

C.C.T.:

Que tous les frais médicaux, y compris les ordonnances de médecins, soient intégralement déduits de l'impôt sur le revenu; que l'on accorde les exemptions suivantes: $\$ 3,000.00$ pour les personnes mariées, $\$ 1,500.00$ pour les célibataires et $\$ 300.00$ pour chaque personne à charge; que l'on inscrive sur la formule TH les cotisations syndicales versées, tout comme c'est le cas pour les fonds de pension. 


\section{C.T.C.C.:}

Les délégués au Congrès de Shawinigan adoptèrent des résolutions demandant que soient exemptés les montants versés en prime "pour la protection en maladie, en accident, au décès, pour un fond de grève et pour les prélèvements faits par les comités paritaires sur les salaires des ouvriers. » On demande également qu'une exemption de $\$ 1,000.00$ d'impôt «soit accordée à celui qui défraie les dépenses pour tout étudiant, aussi longtemps que ses études ne seront pas terminées, quel que soit son âge. On adopta de plus une résolution demandant «que l'impôt sur le revenu sur le temps supplémentaire soit supprimé, dù à la rareté de main-d'oeuvre. »

\section{d) Logement}

Le rapport du Comité du logement du C.M.T.C. adopté à l'unanimité souligne le désappointement du Congrès devant la réponse du Premier ministre du Canada à leur demande touchant l'habitation. Le Congrès continuera ses efforts en vue d'obtenir des habitations à bas loyers. Une résolution demande que le taux d'intérêt pour la construction et l'achat de maisons ne soit pas supérieur à $3 \frac{1}{2} \%$ et que les prêts nécessaires soient consentis par l'entremise de la loi nationale sur le logement.

La politique gouvernementale en matière de logement et la loi nationale sur l'habitation furent critiquées de façon acerbe par nombre de délégués du C.C.T. Une résolution fut même renvoyée au comité des résolutions parce qu'elle n'était pas assez énergique. Cette résolution accusait le gouvernement d'avoir failli à sa tâche de satisfaire à la demande de logements pour ceux qui ne peuvent se construire de maisons. Elle demandait au gouvernement, entre autres choses, de prendre les mesures nécessaires pour répondre aux besoins en matière d'habitation et en particulier de modifier la loi nationale sur l'habitation afin que l'on n'exige qu'un faible déboursé initial et des taux d'intérêts peu élevés; que les prêts soient consentis directement par l'entremise de la Société centrale d'hypothèque et de logement à ceux qui désirent se construire une maison. Dans cette résolution, on demandait également au gouvernement de mettre en oeuvre un vaste programme de construction à loyer modique dans les centres urbains.

Alors que le C.C.T. et le C.M.T.C., en ce qui concerne la question du logement, concentrent leur effort exclusivement sur une action gou- 
vernementale, la C.T.C.C. en plus de faire pression sur le gouvernement, presse ses membres eux-mêmes à agir. Par les résolutions et les recommandations présentées au congrès de la C.T.C.C. ,on peut voir que certaines concernent le gouvernement et d'autres les membres.

Au gouvernement, on demanda de faire bénéficier les salariés des articles 9 et 35 de la loi nationale d'habitation et de faire conclure, à ces fins, une entente entre les autorités fédérales, provinciales et municipales. On lui demanda également de se prévaloir de l'article 35 de la Loi nationale, d'aménager des terrains et de construire des logements familiaux pour fins de location ou de vente. On lui présenta une autre résolution à savoir: la remise en vigueur du prêt additionnel d'un sixième du prêt conjoint aux propriétés. Une dernière demande fut soumise pour que le parlement provincial permette aux Caisses populaires de consacrer $50 \%$ de leur liquidité à des prêts sur des habitations familiales et, que le gouvernement garantisse $50 \%$ de ces prêts.

Comme il a été dit précédemment, la C.T.C.C. demanda à ses membres d'agir et pour ce, on recommanda d'abord qu'une enquête soit instituée afin de connaître la situation des membres au point de vue logement; que soit créée, chez les membres, une caisse d'habitation dont l'argent servirait au financement temporaire de tout plan de construction d'habitation familiale. Une autre demande aux corps affiliés de la C.T.C.C. est de favoriser ou de susciter la formation de compagnie à dividende limité ne dépassant pas $5 \%$ afin de prendre avantage de l'article de la Loi nationale. Cet article prévoit que la société centrale peut consentir à un taux de $3 \frac{1}{2} \%$ des prêts de $90 \%$ de la valeur d'emprunt pour projets d'habitation à bas loyer, lorsque ces prêts sont accordés à ces compagnies à dividende limité ne dépassant pas $5 \%$.

\section{e) Immigration}

De nombreuses résolutions exigeant un contrôle très sévère de l'immigration furent présentées lors du congrès du C.C.T. On y substitua un voeu demandant au gouvernement de créer un comité consultatif de l'immigration comprenant une juste représentation ouvrière, afin de «maintenir une revue constante de la Loi et de son application et de la politique d'immigration. » Ce comité conseillerait le gouvernement sur les changements dans la loi que le gouvernement se propose ou que le comité estime nécessaires. On recommande l'établissement immédiat d'une commission royale chargée d'étudier la loi et son application ou à défaut, un comité parlementaire, afin d'apporter à la Loi ou à son application les modifications nécessaires. 
Les délégués au C.M.T.C. blâmèrent fortement la politique d'immigration actuelle. Principal grief: beaucoup de Canadiens sont sans travail alors que des flots d'immigrants nous arrivent. Le Congrès proteste donc énergiquement «auprès des autorités de l'immigration qui permettent au personnel étranger de travailler au Canda avant de s'être assurées si parmi nos sans-travail il n'y a pas de citoyens canadiens disponibles pour remplir ces emplois. Entre autres choses, le Congrès recommande la revision complète de la Loi de l'immigration et la réduction du flot d'immigration durant l'hiver et les mois de morte-saison. » Enfin on demande la création d'une commission gouvernementale où seraient également représentés les ouvriers, les patrons et le gouvernement. »

\section{f) Assurance-chômage}

Le C.M.T.C. demande que toutes les personnes ayant un emploi rémunéré dans toutes les occupations soient sujettes à la Loi de l'assurance-chômage. On demande en outre que les travailleurs saisonniers soient admissibles aux prestations, aux mêmes conditions que les travailleurs dans les autres emplois, sans être astreints à verser 180 contributions avant d'avoir droit aux prestations. On formula également des voeux pour que soit éliminée la période d'attente et pour que les prestations soient versées aux travailleurs mis à pied durant une grève légale; on recommande que les prestations soient augmentées de $50 \%$.

Les délégués au C.C.T. eurent à se prononcer sur les recommandations du comité du C.C.T. sur l'assurance-chômage. Ce comité recommandait, entre autres choses, d'éliminer les jours non-indemnisables et de réduire considérablement, sinon supprimer toute période d'attente; d'étendre les dispositions de la Loi aux employés d'hôpitaux; d'élargir le domaine des droits syndicaux prévus par la Loi, afin de protéger les travailleurs refusant de franchir les piquets d'une autre union, etc.; d'ajouter un réime d'invalidité-maladie à l'assurance-chômage.

De nombreuses résolutions portant sur la loi furent présentées lors du Congrès de la C.T.C.C. Citons la substance de quelques-unes de ces résolutions.

a) Que les prestations soient augmentées de $\$ 1.00$ par semaine, dans le cas d'un assuré sans enfant, de $\$ 3.00$ si l'assuré a une personne à charge et de $\$ 6.00$ par semaine, sans augmentation de contribution dans le cas d'un assuré ayant 2 personnes à charge. 
b) Que la période de prestations supplémentaires soit prolongée jusqu'au 30 avril.

c) Qu'après avoir complété la période d'attente, tous les jours non compensables soient abolis même dans le cas d'un travailleur qui travaille à temps réduit.

d) Que la période d'attente soit réduite à 3 jours avant de retirer des prestations et que les prestations soient payées dans un délai maximum de quinze jours à compter de la date de la réclamation.

e) Que dans les cas où un employeur dont les employés sont en grève décide de congédier des employés, la loi, dans l'article 39 , permette à ces employés de recevoir des prestations de l'Assurance-Chômage.

De plus lorsque les employés sont en grève, un employeur ne pourrait recourir au Service national du placement, en annonçant sous son autorité. En outre le Service ne pourrait lui référer aucun postulant.

\section{Coût de la vie et contrôle des prix}

Le C.M.T.C. recommande le retour au contrôle des prix et demande que des subsides fédéraux soient versés afin de réduire le prix au consommateur des item principaux de la nourriture.

La C.C.T. déclare: "Il est résolu que le Congrès demande au gouvernement fédéral le rétablissement de la réglementation des prix. 》

\section{Relations ouvrières, salaires, heures et conditions d'emploi}

\section{C.M.T.C.:}

Les délégués du C.M.T.C. demandèrent l établissement d'un Code national du travail afin d'obtenir plus d'uniformité dans la législation ouvrière à travers le Canada. On demanda également l'établissement d'une semaine de cinq jours et de 40 heures, avec salaire minimum national de 85 cents l'heure et le droit, pour le travailleur à deux semaines de congé payé après une année de service. Le travailleur serait rémunéré pour toutes les fêtes statutaires et recevrait double rémunération s il est obligé de travailler ces jours-là.

Le Congrès approuve l'établissement d'un comité permanent chargé des problèmes des fonctionnaires et recommande que ces derniers 
aient le droit d'être certifiés et de négocier des conventions collectives avec leurs employeurs. On demande la modification des lois existantes afin d'interdire aux Conseils des relations ouvrières de "s'ingérer dans les affaires internes des syndicats sollicitant accréditation.» Les délégués demandent également au gouvernement de reviser les barèmes de justes salaires accompagnant les contrats qu'il accorde de façon à obliger l'employeur «à respecter les conventions syndicales en vigueur dans la région. » «On demande également aux organisations ouvrières de I'Est du pays de faire reviser les taux de salaires minima et d'obtenir ainsi plus d’égalité avec ceux des provinces de l'Ouest. »

\section{C.C.T.:}

De nombreuses résolutions furent adoptées au congrès du C.C.T. au sujet du salaire, des conditions de travail et de la législation. On a demandé d'amender l'Acte de l'Amérique Britannique du Nord et de remplacer la législation provinciale par trois nouvelles lois fédérales, dont l'une comporterait l'établissement d'un code national de travail concernant toutes les industries. Une autre légiférerait sur une semaine de 40 heures avec taux obligatoire de surtemps pour les heures additionnelles. Enfin on établirait par une troisième Loi un salaire national minimum approprié dans tout le Canada. On demande au gouvernement d'adopter une politique de pratiques loyales d'emploi, d'abord en sanctionnant une loi nationale sur ce sujet, en insérant des clauses de nondiscrimination dans tous les contrats du gouvernement et en appliquant rigoureusement les dispositions contenues dans la loi d'assurance-chômage visant les activités du Service national de logement.

Le Congrès déplore la tendance dans certaines provinces à circonvenir les droits du travail par l'usage de brefs d'injonction et d'appels devant les tribunaux et il souhaite que l'on prenne tous les moyens possibles pour maintenir ces droits contre les recours abusifs aux tribunaux par les employeurs et le gouvernement.

Les gouvernements, fédéral et provinciaux, devraient de l'avis de cette centrale donner l'exemple en levant les restrictions frappant les travailleurs plus âgés et devraient qualifier de pratique déloyale semblables restrictions de la part d'employeurs privés. Tout comme ce fut le cas du congrès du C.M.T.C., le C.C.T. demande que les syndicats de fonctionnaires fédéraux soient reconnus et qu'ils aient le droit de négocier collectivement avec leurs employeurs par l'entremise du syndicat 
de leur choix. Le même voeu s'applique dans le cas des employés de la Couronne.

La C.C.T. adopta également un projet d'organisation concertée dans le Québec. Une telle campagne était déjà l'un des projets annoncé par le C.M.T.C., lors de son congrès en août dernier. "Seuls les syndicats C.I.O.-C.C.T. », déclare le préambule de la résolution, «peuvent réellement représenter comme ils couvrent les milliers de travailleurs non syndiqués ou médiocrement syndiqués du Québec et leur obtenir des hausses suffisantes de salaire et des conditions de travail plus favorables». Le Congrès est d'avis que le mouvement syndical tout entier de la province de Québec est menacé par «l'activité anti-syndicale»du gouvernement provincial; et il enjoint également au bureau du Congrès de "prendre tous les moyens à sa disposition pour défendre les droits des travailleurs contre le recours injuste aux tribunaux par les employeurs et le gouvernement.

\section{C.T.C.C.:}

Il fut surtout question des problèmes ouvriers de notre province lors du dernier congrès de la C.T.C.C., qui recrute la majeure partie de ses effectifs dans la province de Québec. Différents rapports sur ces sujets furent adoptés par les délégués. Rappelons les passages les plus importants: «L'exercice du droit d'association n'est pas efficacement protégé dans notre province. Les suspensions et les congédiements pour activités syndicales sont trop nombreux.» - «On rémédierait à bien des maux si on créait un comité de relations industrielles à l'Assemblée législative, et si on dressait une liste de noms de magistrats, juges et autres personnes compétentes qui pourraient agir en qualité de présidents des conseils d'arbitrage, lorsqu'il n'y a pas accord entre les parties sur le choix du tiers-arbitre. L'on pourrait aussi choisir ces arbitres parmi les juges de toutes les cours de la Province». Un autre rapport souligne qu' «à cause de l'atmosphère anti-syndicale que l'on s'est plu à développer dans la province depuis quelques années, certains employeurs en profitent pour tenter de se débarasser du syndicat qui groupe leurs ouvriers. »

D'autre part, il est à craindre que par les nombreux délais accumulés devant la Commission de Relations ouvrières ou les tribunaux d'arbitrage, «on en vienne pratiquement à nier leur droit de grève et, partant l'exercice de leur droit d'association». Par exemple «Des employeurs de mauvaise foi ont vu dans le recours aux brefs de prérogative un excellent moyen de prolonger indéfiniment les délais légaux». 
Les travailleurs ont un droit strict au respect et au maintien de leur organisation et un droit moral à la position qu'ils occupaient avant la grève, déclare M. Jean Marchand. Cependant, poursuit le Secrétairegénéral, on veut souvent profiter de la grève pour anéantir ces droits et tuer le syndicat. Alors, on obtient des injonctions contre le piquetage; on obtient la protection de la police pour les briseurs de grève. Les travailleurs se sentent injustement traités. Le secrétaire général ajoute: «nous préférerions, comme le réclame la C.T.C.C. que les injonctions soient complètement abolies dans les cas de grève légale.»

L'expérience avec les tribunaux d'arbitrage est désastreuse et, à moins «qu'une réforme prochaine ne soit entreprise, nous craignons que l'institution elle-même ne soit définitivement compromise». Tout d'abord ces tribunaux devraient accorder toute l'attention voulue aux revendications syndicales et il faudrait que ces tribunaux soient présidés par des gens compétents et désintéressés. Ils devraient avoir « des connaissances générales en économie politique, sur le mouvement ouvrier, sur l'industrie à laquelle se rattache l'entreprise concernée et sur les lois qui peuvent s'appliquer ». De tels candidats sont peu nombreux, aussi « il ne faudrait pas se limiter arbitrairement dans leur choix comme on le fait présentement». De plus, déclare-t-on aux délégués, il faudrait que les décisions soient basées sur des considérations pertinentes à la preuve et au litige. Il faudrait également un plus grand nombre de bons conciliateurs auxquels on donnerait plus de latitude dans l'accomplissement de leur fonction. Ainsi leur prestige en serait fortement accru auprès des parties.

En plus d'adopter le Rapport Moral du Président et celui du Bureau Confédéral dont nous avons souligné quelques points, les membres délégués de la C.T.C.C. votèrent de nombreuses résolutions explicitant les revendications contenues dans les rapports. Des résolutions demandent à ce que soit reconnue la pratique du Droit d'Association, et que l'employé congédié pour activités syndicales soit repris à son ancien emploi avec pleine restitution de ses droits acquis dans l'entreprise et pleine compensation pour perte de salaire. De plus la Commission de Relations ouvrières accomplirait elle-même toutes les procédures nécessaires à cet effet.

"Que des mesures efficaces soient mises en vigueur par la Commission de Relations Ouvrières, pour protéger le droit des ouvriers de s'organiser sans crainte d'intimidation ou de discrémination de la part de l'employeur». Une autre résolution proteste contre les délais alar- 
mants dans l'administration des lois ouvrières », particulièrement dans les procédures d'arbitrage et désire constamment... une réponse urgente et rapide...»

La C.T.C.C. demande également:

a) que la loi soit amendée pour que la durée des conventions collectives soit pour une période d'une durée quelconque, mais inférieure à trois ans.

b) Un amendement à la loi permettant de faire de l'organisation syndicale dans toutes les villes dites «fermées et en forêt »...

c) Que la période de 14 jours qui suit l'arbitrage soit réduite à 7 jours.

d) De limiter le règlement des griefs pour les employés des services publics à 60 jours.

e) Un amendement à la loi permettant d'inclure les surintendants et les contremaîtres comme «salariés » dans les définitions de la loi.

f) Un amendement à l'Ordonnance no 4 réduisant de 54 à 48 heures, les heures de travail des employés du commerce.

g) Que les employés en forêt travaillent de 8 heures a.m. à 5 p.m. avec une heure de repos le midi, et que temps et demi soit payé pour les heures supplémentaires.

h) D'accroître l'aide aux centres d'apprentissage, afin de répondre aux besoins croissants des centres existants.

i) Une pression auprès du Conseil de l'Instruction Publique afin d'obtenir une représentation de la C.T.C.C. au Comité spécial de coor. dination de l'enseignement.

\section{Formation syndicale}

Le C.M.T.C. demande l'établissement d'un collège ouvrier.

Le C.C.T. encourage le développement continu de son département d'Education et on demande une plus grande insistance sur l'histoire du syndicalisme ainsi que sur la structure et les pratiques des syndicats. L'éducation syndicale devrait prendre une plus grande importance dans les syndicats mêmes. On devrait encourager les Conseils et les Fédérations du travail à prendre en main leurs propres programmes d'éducation. 
La C.T.C.C. annonce un manuel d'éducation ouvrière dont le but sera «de poser des bases solides à notre effort de formation syndicale. » Afin d'assurer le progrès, la permanence et l'unité de la formation ouvrière, le service d'Education propose aux syndicats les objectifs suivants:

a) Multiplier les services régionaux d'éducation au sein de chaque Conseil central «qui est l'organisme de notre mouvement le mieux placé pour remplir cette fonction syndicale de première importance».

b) Parfaire au moyen d'écoles d'Action Ouvrière l'étude des problèmes ouvriers abordés dans les cercles d'étude. Répandre l'initiative d'Ecole d'Action ouvrière destinée aux syndiqués féminins.

c) Répéter l'heureuse initiative des sessions d'études spécialisées pour les permanents du mouvement.

d) Déléguer des membres au Collège Ouvrier inauguré à Québec au mois de novembre. ${ }^{+}$

e) Organiser des écoles de délégués de département, «les militants de base du syndicalisme», car ils ont besoin d'une formation spéciale afin de pouvoir assurer la relève du mouvement et d'entraîner la masse.

f) Se procurer le manuel de la Convention Collective de travail à paraître à l'automne.

Le rapport cite avec avantage l'aide considérable et précieuse reçue du Centre de Culture Populaire de Laval dont le but est de contribuer à la formation d'une élite populaire, par tous les moyens à sa disposition et en particulier, en permettant à nombre de professeurs de participer à l'oeuvre d'éducation entreprise par les mouvements syndicaux.

\section{Autres résolutions}

C.M.T.C.:

1) Le congrès exhorte le gouvernement fédéral à faire tout en son pouvoir pour obtenir la paix mondiale par l'entremise des NationsUnies et du Pacte Atlantique.

(4) N.D.L.R. Le Collège Ouvrier remporta un tel succès qu'il est à souhaiter qu'on l'établisse sur des bases permanentes et qu'il puisse fonctionner à l'année. Si le sérieux de «l'étudiant» est un gage de succès, nous sommes convaincu par notre propre expérience qu'un Collège Ouvrier permanent offre de grandes promesses au mouvement syndical. 
2) La télévision devrait être laissée entièrement à Radio-Canada tout comme la Radio.

3) Le gouvernement devrait adopter des mesures destinées: a) à prévenir le chômage; b) à aider les pays sous développés; c) à développer notre commerce extérieur; d) à établir de nouvelles industries là où on développe nos ressources naturelles.

4) On devrait nationaliser toutes les entreprises de téléphone et tous les services téléphoniques.

5) Bannir la littérature obscène ainsi que «les photographies inconvenantes».

C.C.T.:

1) «Les travailleurs du Canada s'engagent à conserver leur appui à l'Organisation des Nations Unies ».. . «La présente réunion s'engage à appuyer l'effort de défense du Canada, mais elle demande que le fardcau du réarmement impose un sacrifice égal à tous ies secteurs de la population canadienne ». Comme la paix et la sécurité universelles dépendent également de l'amélioration des niveaux de vie déplorables en divers pays et régions de l'univers, «il faudra que les pays comme le Canada accordent une assistance économique et technique sur une échelle beaucoup plus grande qu'ils ne le font actuellement» mais cette assistance «ne doit pas servir à soutenir des régimes locaux corrompus. . .» « le Canada devrait venir en aide aux peuples qui luttent encore pour satisfaire leurs aspirations nationales ».

2) Le Congrès verra à lancer une campagne en vue d'organiser les employés de bureau.

3) Le Congrès demande d'augmenter les allocations familiales.

4) La C.C.T. approuve la canalisation du St-Laurent, à titre de projet canadien.

C.T.C.C.:

La Confédération soumet des résolutions pour

1) Obtenir des allocations de maternité et faire augmenter de $50 \%$ les allocations aux mères nécessiteuses.

2) Faire exiger par le gouvernement que les armateurs canadiens fassent construire et réparer leurs navires au Canada, par des chantiers maritimes canadiens. 
3) Amender la loi relative aux rentes de l'Etat et porter le maximum à $\$ 2,400.00$.

4) Baser l'évaluation municipale sur la propriété, pour fins de taxation non pas sur la valeur ou le coût de la propriété, mais d'après sa valeur locative.

Remettre $50 \%$ du revenu de la vente des licences dautomobile aux municipalités où résident les automobilistes de mème que $50 \% \mathrm{du}$ revenu sur la taxe de la gazoline vendue dans les limites de ces municipalités.

5) Augmenter les allocations familiales de $50 \%$, et les maintenir dans le cas des enfants aux études, tant que dureront les études.

6) Accorder le droit de vote à toute personne « dès l’àge de 18 ans ».

7) Former un comité de stratégie de la C.T.C.C., dont les objectifs seraient: a) faciliter un meilleur échange d'information; b) après avoir étudié la conjoncture économique et sociale, orienter le travail revendicatif des fédérations; c) orienter la campagne d'organisation de nouveaux syndicats ou de nouveaux secteurs industriels.

Ce comité ferait des recommandations, mais ne pourrait lier les différents corps du mouvement. Il serait purement consultatif.

Nous venons de voir les résolutions et recommandations des diverses organisations ouvrières au Canada, C.M.T.C., C.C.T. et C.T.C.C. qui ont été soumises lors de leur réunion annuelle respective pour 1952 . On peut constater par là l'importance de ces congrès pour les membres euxmêmes, en leur permettant d'unir leur force pour poursuivre leurs fins communes, pour les chefs, en leur donnant une nouvelle occasion favorable d'entrer en contact direct avec leurs membres et, pour tous, en faisant connaître les positions et tendances du syndicalisme au Canada en $1952 . *$

* N.D.L.R.-Dans son étude l'auteur réfère aux documents suivan's:

The Canadian Unionist, Vol. XXVI, No. 9, September 1952, No. 10, October 1952, Official Journal of the Canadian Congress of Labour, Hull, Que. - La Gazette du Travail, Vol. LII, no 9, septembre 1952, publié par le Ministère du Travail, Ottawa. - The People's School, Bulletin No. 1, October 12, 1952, Bulletin No. 2, October 19 1952, published by St. Francis Xavier Extension Department, Antigonish, N.S. - Procèsverbal, trente et unième session du Congrès de la C.T.C.C., ShawiniganFalls, P.Q., 1952. - The Trades and Labor Congress Journal, Vol. XXXI, No. 9, September 1952, No. 10, October 1952, official magazine of the Trades and Labor Congress of Canada, Ottawa. 